\title{
Effect of Buckling Modes on the Fatigue Life and Damage Tolerance of Stiffened Structures
}

\author{
Carlos G. Dávila ${ }^{1}$ \\ NASA Langley Research Center, Structural Mechanics and Concepts Branch, Hampton, VA, 23681 \\ Chiara Bisagni ${ }^{2}$ \\ University of California San Diego, Department of Structural Engineering, La Jolla, CA, 92093 \\ and \\ Cheryl A. Rose ${ }^{3}$ \\ NASA Langley Research Center, Structural Mechanics and Concepts Branch, Hampton, VA, 23681
}

The postbuckling response and the collapse of composite specimens with a co-cured hat stringer are investigated experimentally and numerically. These specimens are designed to evaluate the postbuckling response and the effect of an embedded defect on the collapse load and the mode of failure. Tests performed using controlled conditions and detailed instrumentation demonstrate that the damage tolerance, fatigue life, and collapse loads are closely tied with the mode of the postbuckling deformation, which can be different between two nominally identical specimens. Modes that tend to open skin/stringer defects are the most damaging to the structure. However, skin/stringer bond defects can also propagate under shearing modes. In the proposed paper, the effects of initial shape imperfections on the postbuckling modes and the interaction between different postbuckling deformations and the propagation of skin/stringer bond defects under quasi-static or fatigue loads will be examined.

\section{Introduction}

C OMPOSITE stiffened panels used for the construction of aerospace structures can sustain loads far in excess of their buckling loads. The collapse of stiffened panels is generally observed deep into the postbuckling range and is usually due to the interaction between the postbuckling deformations and local damage modes such as skin/stringer disbonding. For this reason, the ability to predict the collapse load and the mechanisms involved in the failure is crucial for the assessment of damage tolerance and the rational design of these structures. The present work consists of an examination of the postbuckling deformations exhibited by Single Stringer Compression Specimens (SSCS).

In the following section, the results of three test campaigns conducted to evaluate the damage tolerance and fatigue life of SSCS are presented[1-4]. Then, the effects of initial shape imperfections on the buckling mode shape and the interaction between the postbuckling deformations and the propagation of an embedded defect are discussed.

\footnotetext{
${ }^{1}$ Aerospace Research Engineer, Structural Mechanics and Concepts Branch, NASA LaRC, Hampton, VA, carlos.g.davila@nasa.gov, AIAA Member.

${ }^{2}$ Professor, Department of Structural Engineering, 9500 Gilman Drive, cbisagni@eng.ucsd.edu, AIAA Associate Fellow.

${ }^{3}$ Aerospace Research Engineer, Structural Mechanics and Concepts Branch, NASA LaRC, Hampton, VA, cheryl.a.rose@nasa.gov, AIAA Member.
} 


\section{Single Stringer Compression Specimen (SSCS)}

The Single Stringer Compression Specimen (SSCS) was developed by the authors to study the response and the failure of a multi-stringer panel loaded in compression [2,3]. The SSCS represents an intermediate level of complexity between coupon-level specimens and structural components that exhibits most of the challenges that characterize the assessment of the damage tolerance response of a more complex multi-stringer panel. Advantages exist from the experimental point of view, due to low manufacturing and testing costs, and from the numerical point of view, due to the moderate computational model size requirements.

The SSCS studied is composed of one stringer, and extends transversely from half bay to half bay with a total width of $150 \mathrm{~mm}$. The dimensions of the SSCS are shown in Fig. 1. The skin consists of an 8-ply quasi-isotropic laminate with a stacking sequence of $\left[45^{\circ} / 90^{\circ} /-45^{\circ} / 0^{\circ}\right]_{\mathrm{s}}$ and a total thickness of $1 \mathrm{~mm}$. The stringers are composed of a 7-ply laminate with a symmetric stacking sequence of $\left[-45^{\circ} / 0^{\circ} / 45^{\circ} / 0^{\circ} / 45^{\circ} / 0^{\circ} /-45^{\circ}\right]$, which results in a total thickness of $0.875 \mathrm{~mm}$. The skin and stringer are co-cured and the material is IM7/8552 tape. An initial bond defect is introduced in some specimens by placing a Teflon film between the skin and one of the stringer flanges, as shown in Fig. 1. Teflon film lengths of 20 and $40 \mathrm{~mm}$ were evaluated.

It can be observed that the longitudinal edges of the SSCS are free, while the corresponding locations of the multi-stringer structure are subjected to the restraining effect of the surrounding structure. This difference of boundary conditions has a significant effect on the shape of the buckling mode, which in turns plays an important role on the interlaminar and intralaminar damage mechanisms in the postbuckling range. Parametric studies were performed to identify the dimensions of the SSCS that ensure a stress distribution similar to that observed in the multi-stringer panel. The studies for a specific multi-stringer design revealed that the skin/stiffener interaction and the corresponding maximum stress failure index distribution can be correctly represented in the proximity of the flange of the stringer using a specimen $240 \mathrm{~mm}$-long, and that the SSCS provides a valid means for assessing the damage tolerance of the multi-stringer panel.

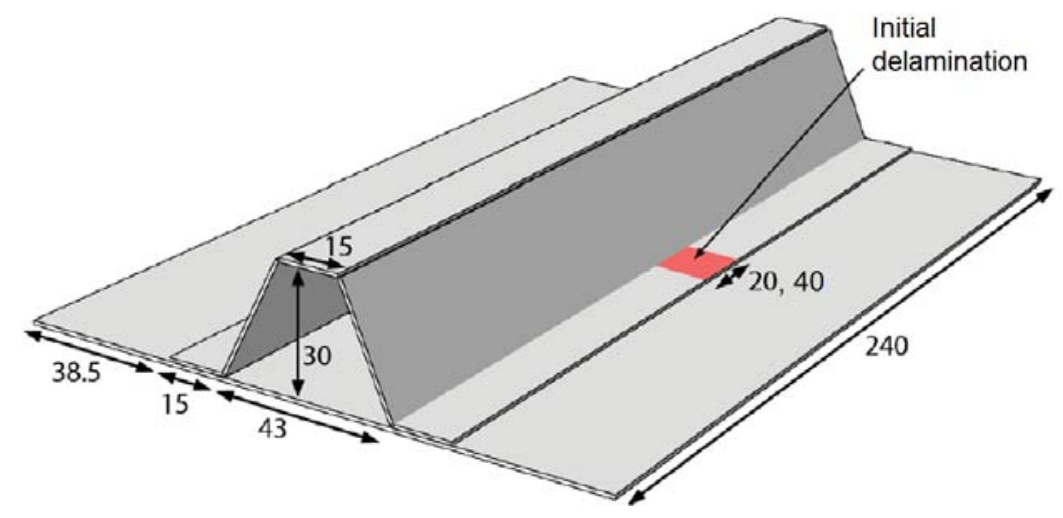

Figure 1. Dimensions of the SSC specimen (in mm).

\section{A. Finite Element Model}

The finite element model of the SSCS consists of shell and cohesive elements and is analyzed with the Abaqus/Std v6.10 finite element code. The skin is composed of a layer of S4 shell elements and the stringer is modeled with another layer. Wall offsets are applied so that the nodes for both shell layers are coincident in the flange sections. The model is defined parametrically, so that any dimension of the specimen and the mesh refinement can be modified instantly. The elements have a typical size of $0.8 \mathrm{~mm}$, and the total number of degrees of freedom is approximately 710,000 .

The major damage modes observed experimentally, skin-stringer separation and intralaminar damage, are taken into account by the model. The skin-stringer separation failure mode is modeled by placing a layer of cohesive elements between the skin and the stringer along the flanges of the stringer. These elements can represent mixedmode delamination growth with a damage evolution law specified as a bilinear constitutive equation. In the case of the specimen with a Teflon insert, pre-damaged cohesive elements are placed in the area of the Teflon insert.

The initiation and evolution of intralaminar damage is studied using a continuum damage model. The damage mechanisms occurring in the fiber and the matrix are represented by a set of scalar damage variables. Damage 
activation functions based on the LaRC04 failure criteria are used to predict the different failure mechanisms occurring at the ply level [5].

The analysis of the SSCS consists of a thermal analysis followed by a mechanical load. The thermal analysis accounts for the residual thermal stresses that result from contraction between the curing temperature to room temperature. The boundary conditions applied during the thermal step consist in constraining one of the two ends of the specimen while allowing free expansion in the longitudinal direction. The mechanical load consists of the application of an end shortening displacement. The end tabs of the specimens are simulated by constraining the translational degrees of freedom of the corresponding nodes.

To avoid convergence difficulties in the load incrementation procedure, an implicit dynamic solution was adopted. Details about the model and material properties used can be found in Refs. [2, 6]

\section{B. Experimental Program}

The present investigation relies on the results of three test campaigns performed by the authors. The first one, conducted at the Politecnico di Milano, was used mainly to verify the design of the specimen and the end potting, and to develop a progressive damage analysis capable of predicting the initiation and propagation of skin/stiffener debonding as well as the intralaminar damage mechanisms that cause crippling of the stringer [2]. The second test campaign was conducted at the NASA Langley Research Center, and was distinguished from the first by an extensive use of digital image correlation (DIC) to monitor the formation and evolution of the postbuckling deformations[3]. The specimens were subjected before the tests to high-resolution ultrasonic scanning (UT) to ensure that they were free of manufacturing defects. Their initial deformations were also carefully measured in a Coordinate Measuring Machine (CMM), as shown in Fig. 2. The final test campaign, which was also conducted at the NASA Langley Research Center, focused on the evolution of the fatigue life of SSCS with two sizes of embedded defects[4]. DIC, in-situ UT scanning and passive thermography were used during the fatigue loading.

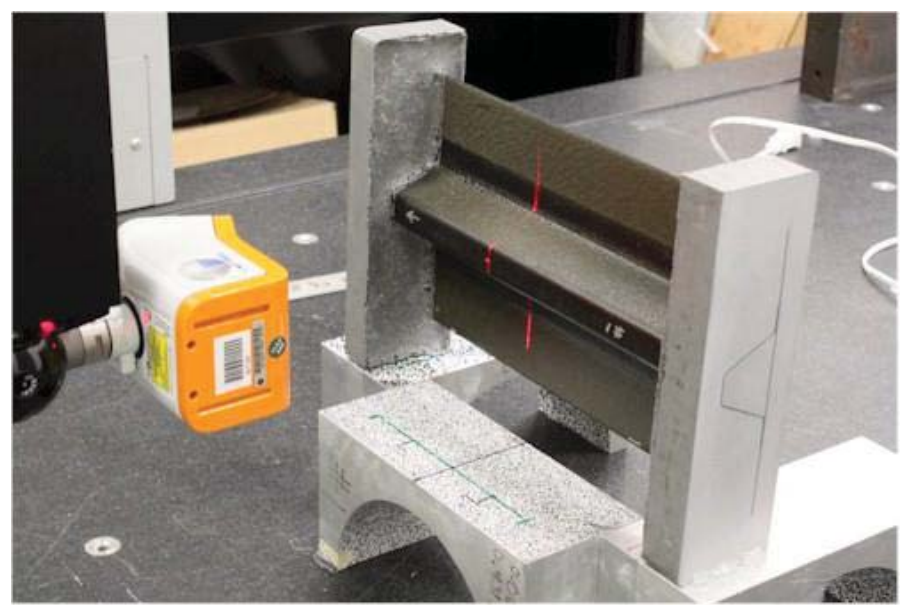

Figure 2. Laser scanning of SSC specimen with Coordinate Measuring Machine (CMM)

\section{Response of SSCS}

The response of the SSCS is characterized by several phases. The response phases for a nominally pristine specimen at different load levels are illustrated in the analysis results shown in Fig. 3. In the first phase of the analysis, the specimen exhibits a linear response with a single-wave out-of-plane deformation mode along the free edge of the skin (Point A). At an applied load of $7.5 \mathrm{kN}$, Point B corresponds to the first buckling load, which affects only the skin. Three half-waves are observed along the longitudinal direction. Stringer buckling is predicted at a load of $23.6 \mathrm{kN}$ (Point C). Shallow buckles become visible on the two webs of the stringer which form into a series of inclined half-waves. The crown of the stringer remains stable, and the stringer can continue sustaining higher loads. At point $\mathrm{D}$, which corresponds to $38.5 \mathrm{kN}$, skin/stringer separation initiates near the mid length of the stringer at a location on a flange where the out-of-plane displacements in the direction away from the stringer are the largest (blue areas in Fig. 3b). The initiation of separation also corresponds with the peak load of the specimen. Point E represents a state past peak load. Here the specimen has collapsed, and the skin and stringer are separated over a 
large region of the flanges. The skin is no longer supported by the stringer and its deformed shape is composed of a single irregular longitudinal half-wave. Simultaneously, the load is transferred to the stringer, which fails instantly by crippling across its entire cross-section.

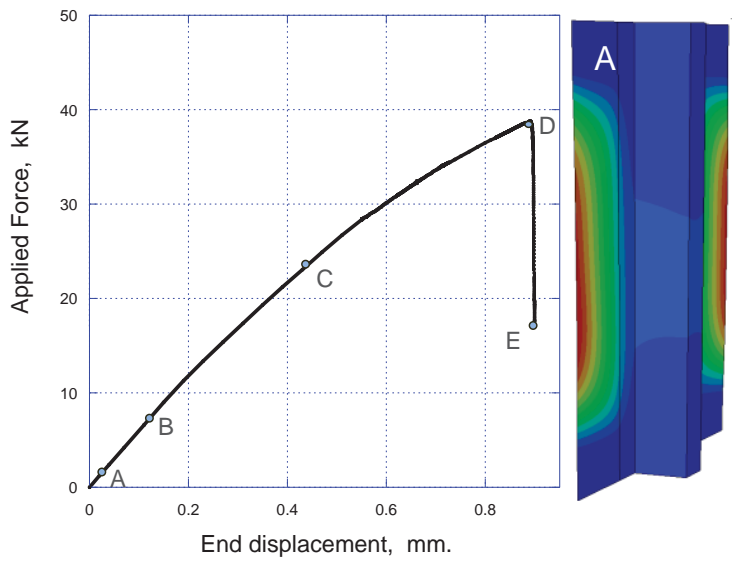

a) load-displacement curve
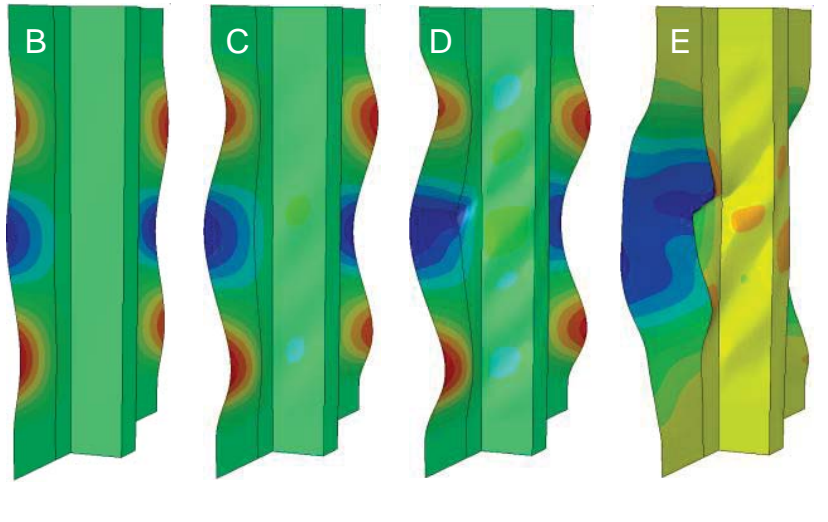

b) out-of-plane response.

Figure 3. Predicted load-displacement and out-of-plane response of the nominally pristine specimen.

\section{A. Buckling Modes}

The most common skin postbuckling mode seen in the experiments, which is also the one predicted by analysis, is the one shown previously in Fig. 3b [3]. This mode is composed of three half-waves along the free edges of the skin with the upper and lower half-waves directed towards the stringer, and the central half-wave directed away from the stringer. This skin buckling mode does not necessarily occur symmetrically on both sides of the stringer, as can be observed on the left side of Specimen \#2 (Fig. 4b) and the right side of Specimen \#3 (Fig. 4c). This mode is highlighted with a solid grey outline on Fig. 4. It can also be observed that a number of other postbuckling modes are possible, with either two, three or four half-waves along the length. It can be observed that the left side of Specimen \#3 is the only one that exhibits a mode with only two half-waves, as highlighted with a long-dash grey line. All the remaining specimens exhibit modes with four half-waves, as highlighted with a short-dash grey line. The symbol " $T$ " on a white background indicates the position of the Teflon implant in each specimen, if present. A tall white background box indicates a 40 -mm insert, and a shorter one indicates a 20 -mm insert.

The signs of the amplitudes can also vary among specimens, as for instance in Specimens \#1, \#5, and \#6, where the three-half wave modes highlighted with the solid black line outline are of opposite sign compared to those discussed above. Specimens \#1 and \#6 exhibit this skin buckling pattern on both sides of the stringer, while this response is present only on the left side in Specimen \#5.

The maximum out-of-plane displacement measured among the specimens is equal to $5 \mathrm{~mm}$ in the direction opposite to the stringer, and $3.5 \mathrm{~mm}$ in the direction of the stringer.

In the central part of the skin, in the area under the stringer, the SSC specimens present modes with smaller waves. These postbuckling modes originate at applied loads that are higher than those that cause buckling of the free edges of the skin and they are characterized by higher numbers of half-waves (between four and seven). 


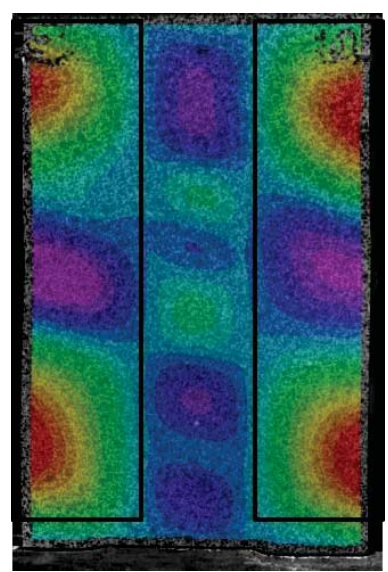

a) Specimen \#1

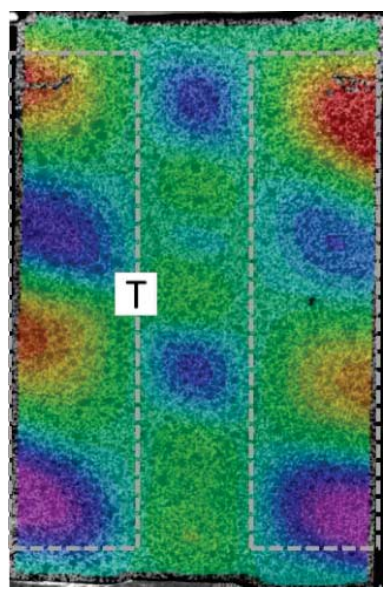

d) Specimen \#4

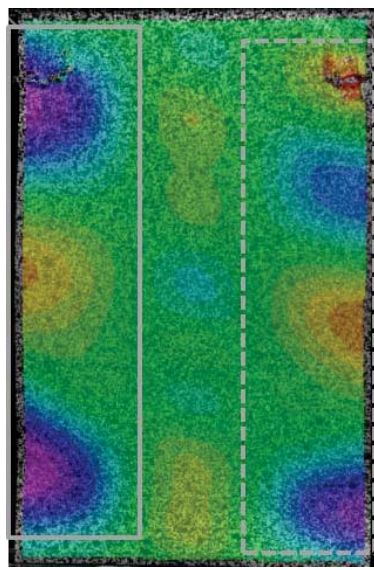

b) Specimen \#2

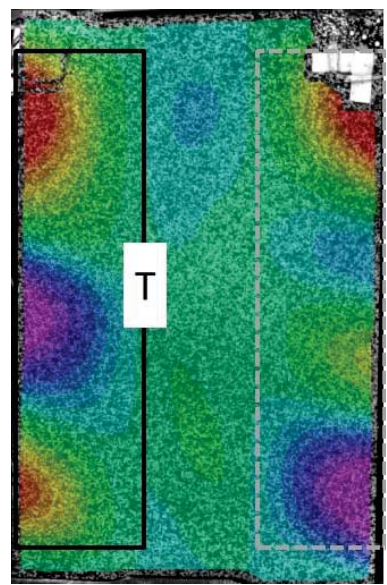

e) Specimen \#5

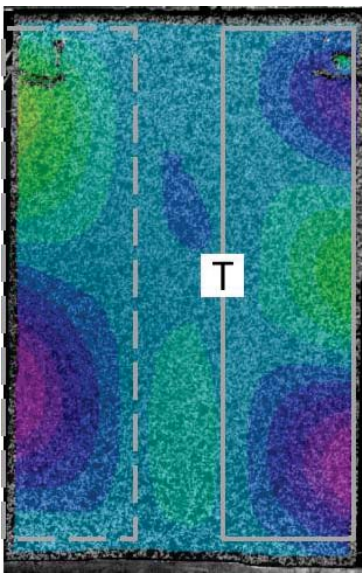

c) Specimen \#3

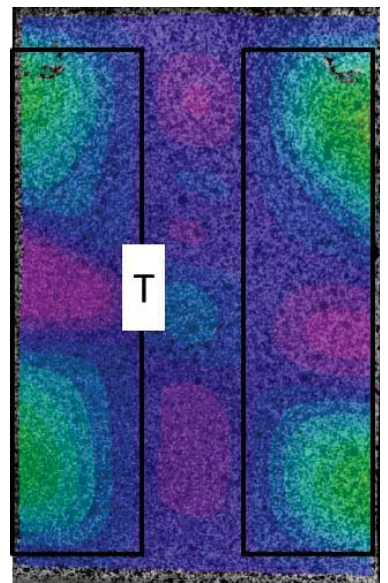

f) Specimen \#6.

Figure 4. Postbuckling modes recorded by digital image correlation for 6 specimens [3].

\section{B. Influence of initial geometric imperfections on the postbuckling mode}

It is fundamental to understand the effects of initial imperfections on the postbuckling response, as different postbuckling mode shapes can affect the initiation and propagation of delamination. Detailed assessments of initial shapes of the specimens were conducted and reported in Ref. [3]. The data recorded includes stringer alignment, curvature of the panel, angle of twist, misalignment angles between loading surfaces, differences in free length of the panel, etc. However, given the limited number of specimens that were tested, an initial attempt to find direct associations between any measured characteristics of the initial shape and a particular postbuckling mode did not lead to clear conclusions. Consequently, a numerical investigation of the effect of imperfections caused by manufacturing and residual thermal strains was conducted.

To investigate the effect of initial geometric imperfections on the buckling mode shape, a finite element model of the SSC specimen previously developed was used. The analyses were conducted in two load steps. In the first step, only thermal loads are introduced. This step captures the initial deformation of the specimen that results from a thermal cool-down. To eliminate rigid body modes, a limited number of boundary conditions that do not generate reaction loads are applied.

The second step of the analysis consists of the mechanical load. The boundary conditions representing the end shortening of the loading platen and those representing the fixed platen are activated. The postbuckling response of the resulting model consists of three half-waves along the free edge, as was shown in Fig. 3b).

Since the model accounts for the residual thermal deformation, which represents the bulk of the initial geometric "imperfection", there is no direct way to apply the measured imperfections to the finite element model. Ideally, a set 
of initial imperfections would have to be estimated such that they produce the desired cumulative imperfections after the thermal loads are applied. However, there is no obvious way to estimate these initial imperfections. Consequently, a study of initial imperfections was conducted by performing parametric analyses in which artificial imperfections associated with an eigenvalue analyses were introduced. The initial imperfections were obtained by applying various linear combinations of the first three eigenmodes and scaling the result to an amplitude of $1 \%$ of the skin thickness. The resulting imperfections were then applied as a perturbation of the initial finite element mesh.

It was observed that different initial imperfections and different thermal curing cycles can change the postbuckling mode. Some examples are shown in Figure 5. In particular, the analysis with a thermal load, with or without initial imperfections, gives a postbuckling mode with three half-waves. However, in the model without imperfections the skin's free edges at the specimen mid-length pull away from the stringer (negative W in Fig. 5a), while the analysis with imperfections predicts the opposite deformation (positive W in Fig. 5b). Both modes were also observed experimentally, as shown in Fig. 5, where the displacement field recorded by the digital image correlation is overlapped on the analysis results. Fig. 5c also shows that different postbuckling modes can be obtained by doubling the thermal loads, in which case four half-waves are obtained along the free edge of the skin. This four-half wave mode was also among those observed experimentally. Some selected test results were superposed over the analysis results in Fig. 5 to show that different initial imperfections result in modes seen in the experiments. However, the analyses lack the predictive capability needed to use measured imperfections to predict the postbuckling response.

Although it is difficult to correlate the real coordinate measurements with the postbuckling mode, the results obtained demonstrate that minor imperfections caused by manufacturing and residual thermal strains can influence the postbuckling mode and may cause differences in the experimental results. These differences in the postbuckling mode can also affect the initiation and propagation of the skin/stringer delamination, as they change the local load distribution, especially in the area close to the stringer. However, additional work is needed to predict the correct postbuckling mode based on measurements of the initial imperfections recorded before the tests.

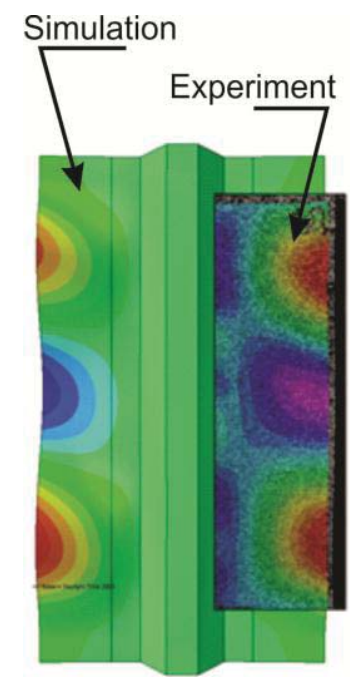

a)

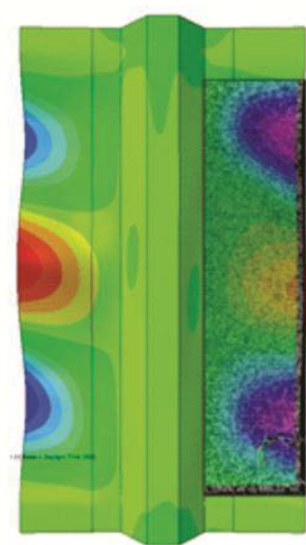

b)
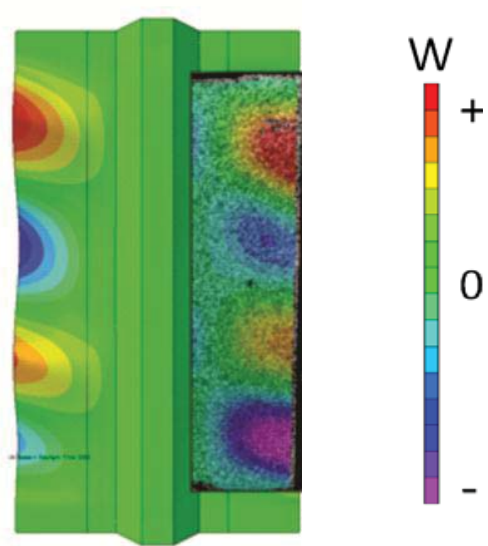

c)

Figure 5. Postbuckling modes obtained with finite element analyses compared with experimental measurements: a) Thermal analysis with no imperfection; b) Thermal analysis with $0.01 \mathrm{~mm}$ imperfection; c) 2X Thermal analysis with $0.01 \mathrm{~mm}$ imperfection.

\section{Effect of Postbuckling Mode on Debonding Mechanism}

The experimental results indicate that the collapse of the SSCS under quasi-static loads is always sudden and catastrophic. No significant damage propagation is observed before final collapse. However, it was observed that the collapse load was influenced by the mode of postbuckling deformation. In the following sections, some of the differences between three of these observed failure modes are discussed. 


\section{A. Symmetric Pullout Mode (pristine specimen)}

The postbuckling deformation of the pristine SSCS model without imperfections is shown in Fig. 6. As expected, the most pronounced deflections are found along the edges of the skin, and minor deflections are observed on the stringer (not shown). Smaller half-waves are also visible in the skin under the stringer (white arrows in Figs. 6b and 6c). When the load is increased, a transition of the postbuckling deformation under the stringer occurs, as observed in the changes between Figs. $6 \mathrm{a}$ and $6 \mathrm{~b}$. The sudden mode jumping in the skin under the stringer determines the redistribution of the internal forces and leads to the immediate delamination between the skin and the stringer flange. This mode of failure is dictated by a sudden mode change and is therefore nearly independent of the properties of the skin/stringer interface.

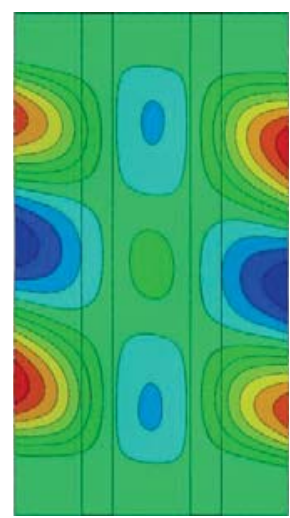

a) postbuckling at $35 \mathrm{kN}$

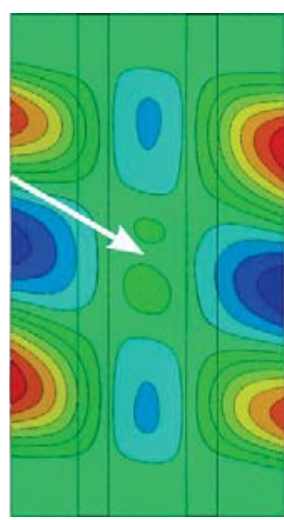

b) mode jumping at $37 \mathrm{kN}$
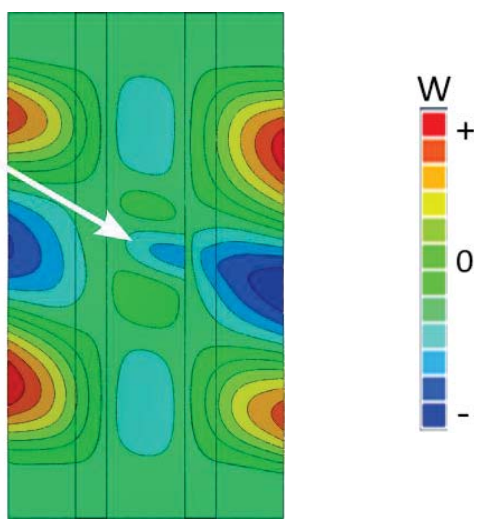

c) delamination at $39 \mathrm{kN}$

Figure 6. Predicted out-of-plane-deflections (view without stringer):

\section{B. Unsymmetric Pullout Mode (SSCS with 40-mm insert)}

The unsymmetric pullout mode initiates as a three-wave symmetric mode in which the skin deflects towards the stringer and, therefore, tends to close interfacial defect, which is on the right of the stringer In Fig. 7. However, at a load of $99 \%$ of the collapse load, the sign of the buckling mode on the side of the stringer with a defect reverses suddenly. This mode reversal induces large peel stresses between the skin and the stringer flange in the vicinity of the initial defect. Shortly thereafter, a delamination between the skin and the stringer tunnels through both stringer flanges (Fig. 7c), causing a sudden failure of the specimen. In the test shown in Fig. 7, the stringer did not cripple and the specimen retained $70 \%$ of the failure load.

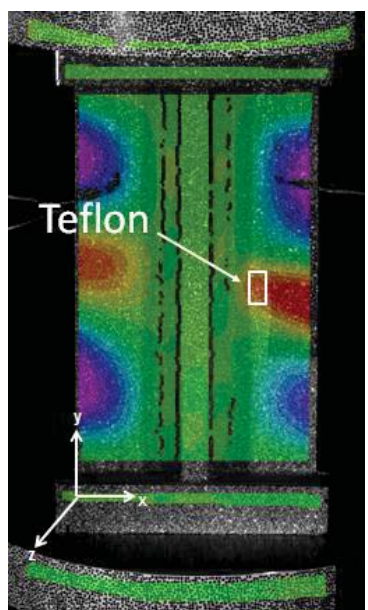

a) Symmetric response at $28.5 \mathrm{kN}$

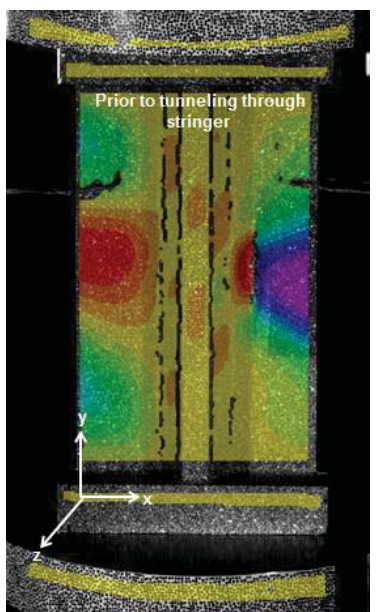

b) Teflon opens at $28.9 \mathrm{kN}$

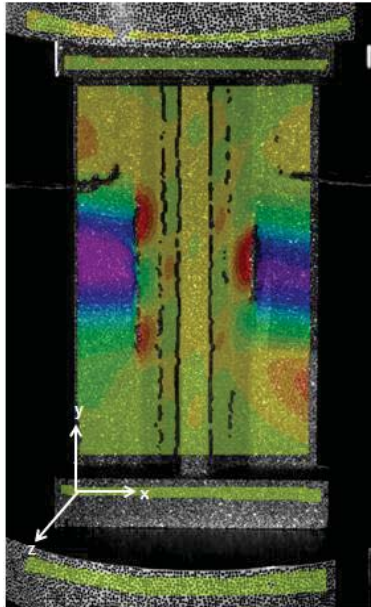

c) Tunneling at $21.1 \mathrm{kN}$

Figure 7. Experimental out-of-plane deflections showing the reversal of the skin deformation next to the stringer and subsequent tunneling of delamination under stringer. 


\section{Shearing Postbuckling Mode}

The propagation of skin/stiffener disbonds can also be driven by shearing deformations. For instance, the embedded Teflon film in Fig. 4d is at an inflection point between two opposite out-of-plane half waves. At this inflection point, the interlaminar shear peaks, which can cause a disbond of the stringer, even without any mode I component. The final version of this paper will include a thorough examination of the difference between the collapse loads of specimens in which the propagation of an interface defect is driven by a shearing mode or a peel mode.

\section{Summary}

The results of several experimental campaigns based on using the SSCS to study the effect of postbuckling mode on the collapse of stiffened panels confirm that nominally identical specimens can exhibit different postbuckling responses. In addition, it is clear that different postbuckling modes have different effects on the propagation of a defect intentionally introduced between the stringer flange and the skin.

Although the present abstract describes only the results of quasi-static tests, data on the postbuckling deformations under fatigue loading have been recorded and are being processed. The full paper will describe the effect of these postbuckling deformations on the fatigue life of the SSCS.

A thorough understanding of the interactions between these postbuckling deformation modes and the collapse of a postbuckled panel will lead to more efficient designs that can safely operate at loads well in excess of the initial buckling load of the panel.

\section{References}

1. Bisagni C, Vescovini R, Dávila CG (2010) Assessment of the Damage Tolerance of Postbuckled Hat-Stiffened Panels Using Single-Stringer Specimens. Proc 41st SDM Conf, Orlando, FL.

2. Bisagni C, Vescovini R, Dávila CG (2011) Single-Stringer Compression Specimen for the Assessment of Damage Tolerance of Postbuckled Structures. J Aircraft. 48.(2).

3. Bisagni C, Dávila CG (2014) Experimental Investigation of the Postbuckling Response and Collapse of a SingleStringer Specimen. Compos Struct. 108.(0): 493-503.

4. Bisagni C, Dávila CG, Rose CA, Zalameda JN (2014) Experimental Evaluation of Damage Progression in Postbuckled Single Stringer Composite Specimens. Proc 29th American Society of Composites Technical Conference

5. Maimí P, Camanho PP, Mayugo JA, Dávila CG (2007) A Continuum Damage Model for Composite Laminates: Part II - Computational Implementation. Mech Mater. 39.(10): 909-919.

6. Vescovini R, Dávila CG, Bisagni C (2012) Simplified Models for the Study of Postbuckled Hat-Stiffened Composite Panels. NASA/TM-2012-217336, Hampton. 